\title{
Article \\ CFD Simulation and Microscopic Study of a Solitary Spherical Porous Adsorbent
}

\author{
John White \\ School of Engineering, Birmingham City University, Birmingham B5 5JU, UK; john.white@bcu.ac.uk
}

\begin{abstract}
Modelling water vapour flow, heat transfer and porosity in porous adsorbent is somewhat challenging simulation problem. Primary macroscopic water vapour flow models, such as Darcy's law, fail to predict the pressure drop entirely correctly for the reason that many of flow parameters not considered because of the simplifications that remain made for the multi-scale structure of the porous adsorbents. For one to develop a good physical understanding of such water vapour flows and the accuracy of existing 3D simulation models, there is a need for some accurate 3D geometry to be studied. This present work describes two-phase water vapour flow and adsorption/desorption performed on porous adsorbent by a Dynamic vapour sorption (DVS). The CFD simulation results are associated with experiments results. It is decided that for such complex porous adsorbent CFD simulation problems the use of COMSOL Multiphysics and SolidWork flow simulation will be utilised.
\end{abstract}

Keywords: Packed beds; Thermal heat; Porosity effect; Thermal contact resistance

\section{Introduction}

Porous adsorbent packed beds have a variety of applications in thermal heat systems. One of the main characteristics of porous adsorbent packed beds is the unique secure surface area to volume. This property is suitable for applications such as adsorption cooling, desorption drying, water and gas filtration, reactor dust collection, and exhaust catalytic and non-catalytic reactors. One essential motivation to study a porous adsorbent packed bed is to regulate the significant operating cost of the pressure drop through the packed adsorbent bed. [1].The thermal heat conductivity of full beds is not isotropic. It is thus difficult to convey a simulation model that completely describes their thermal heat conductivity. Nevertheless, the arrangement of a porous adsorbent packed bed can be modelled assuming asymmetrical adsorbent packing. An asymmetrical adsorbent packed bed is one in which different arrangement of adsorbent spheres uniform in size, is repeated throughout the porous adsorbent bed. Consequently, a representative "number of primary cell" can represent the entire irregular random packing adsorbent bed[18-21].

There are three such types usually considered for porous adsorbent packed beds as can be seen in figure 1: (a) simple cubic (SC), (b) body centre cubic (BCC), and (c) face centre cubic [2]. Revealed that the FCC and the SC are packing allows upper and lower bounds on the thermal heat conductivity of a randomly porous packed bed occupied with a two-phase fluid, separately. Therefore, in this study, the thermal heat of the FCC preparations are considered. 


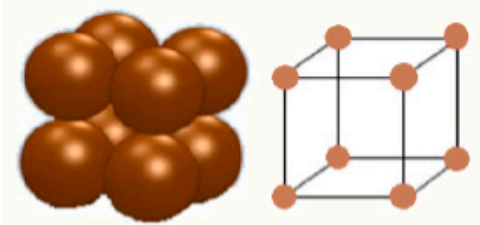

(a) Simple cubic (SC)

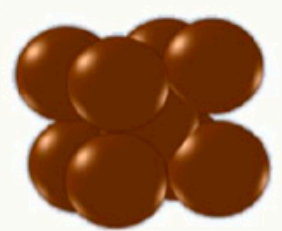

(b) Body centered cubic (BCC)

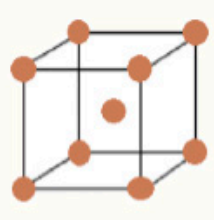

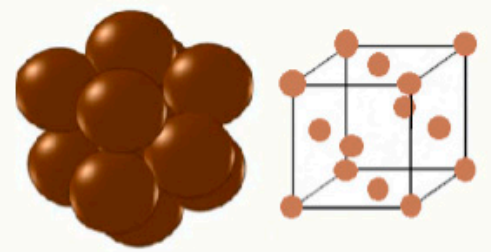

(c) Face centered cubic (FCC)
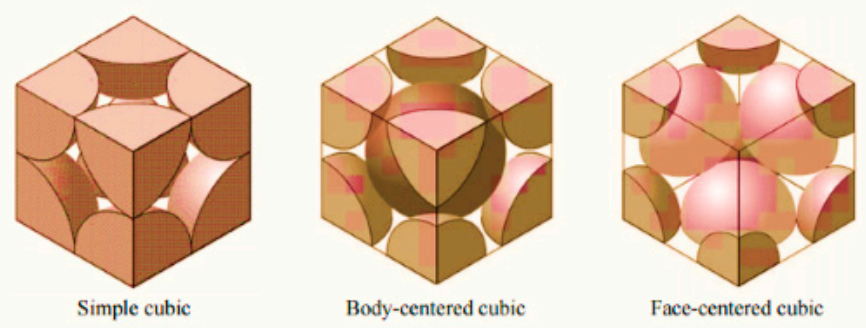

Figure 1 randomly porous packing

The trends of the irregularly packed beds (bounds) can be used to investigate the effect of vital parameters involved in real random packed adsorbent beds.

Several simulation studies were applied for the prediction of thermal heat conductivity of packed adsorbent beds filled with porous adsorbent. The existing simulation models can be categorised into two core groups. The first is CFD numerical models, e.g., finite element methods (FEM) which use the three-dimensional method by dividing the bed into numerous cells with temperature and heat flow corresponding at their boundaries. It is a combined thermal and mechanical three-dimensional simulation analysis that makes the FEM modelling exceedingly expensive from the simulation point of view [3]. Therefore, the thermal contact resistance (TCR) of porous adsorbent must be provided for the software as boundary conditions separately[6,10-22].

The second group is the finite volume method (FVM)[10,18]. This simulation technique breakdown the simulation problem into separate conduction pathways the contact region between adsorbent spheres the water vapour layer between porous adsorbent spheres, etc. The conductivity of the bed is measured as a series/parallel combination of the specific heat resistances of these paths. The advantage of the (FVM) method is that it allows one to calculate with ease the adsorbent packed bed parameters and properties. Different methodologies have been in use by researchers developing CFD simulation model.

\section{The adsorbent Porosity}

Porosity in an adsorbent is defined as the ratio between the adsorbent voids volume and the total volume of the porous adsorbent, also known as the void fraction [9, 11, 16-18]. It is also defined as one minus the adsorbent density $\delta$ and is the most straightforward parameter for characterising the microstructure in a porous adsorbent matrix. Porosity is given as follows:

$$
\begin{gathered}
\varepsilon=1-\delta \\
\varepsilon=\frac{\text { Vvoid }}{V \text { Total }}=1-\frac{\Sigma \text { A Solid }}{\text { ATotal }}
\end{gathered}
$$




\title{
2.1. Adsorbent porosity types
}

When considering adsorbent porosity, it is critical to know what kind of porous you are studying. The International Union of Pure and Applied Chemists standards (IUPAC) there are three types of pore size systems related with porous adsorbents these are[2,5,10]. Microporous, smaller than $2 \mathrm{~nm}$, Mesoporous, between 2 and $50 \mathrm{~nm}$ and Macroporous, longer than $50 \mathrm{~nm}$. Macropores: greater than the usual mean free path length of standard fluid. Bulk diffusion and viscous flow. Mesopores: Same order or smaller than the average free path length. Knudsen diffusion and surface diffusion. Multilayer adsorption and capillary condensation contribute. Micropores: Pore size comparable to the molecules. Activated transport dominates as seen in figure.2.

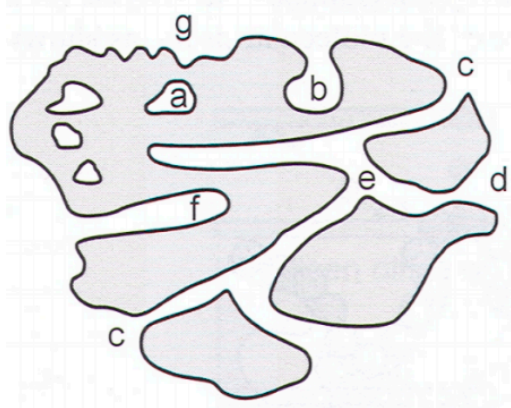

\author{
Accessibility: \\ a: closed pores \\ b,c,d,e,f: open pores \\ b, f: blind pores (dead-end or saccate) \\ e: through pores \\ Shape: \\ c:Cylindrical open \\ f:Cylindrical blind \\ b: ink-bottle-shaped \\ d: funnel shaped \\ g: roughness
}

Figure 2 The influence of the variation of the porosity in a porous adsorbent is considered of great importance in the calculation of the pressure drop, flow and heat transfer characteristics in adsorbent $[2,5,10]$.

\section{Adsorbent Permeability and Porosity}

The permeability of a porous adsorbent can't be defined regarding it porosity and a typical pore size without making an allowance for the pore size distribution. The pore size distribution affects the water vapour flow configuration for the reason that the vapour flow in a given pore does not only depend on the size of the pore being considered, but also on the size of the pores in the opposite direction. Therefore, the measured permeability and the CFD simulated one are different, where the simulated is typically higher than the estimated. It is predominantly problematic to create a permeability simulation model based only on the porosity [8,10,16-18]. It is commonly known that in most porous adsorbent liquid separation processes the vapour flow characterisations through porous adsorbent are important and the anisotropy categorisation can affect the permeability results [16]. Porous adsorbent adsorption of water vapour quality is affected by the pores and their size.

\subsection{Porosity and Cell and Pore in Adsorbent Structure}

In this present study, the internal structure (or microstructure) of the porous adsorbents are represented by cells and pore as seen in Figure 3 and 4. The interior fabric of the porous celled is an arrangement of cells connected to each other by tendons. Each cell has 11 ports or windows as seen in Figure 3 and 4. The pore's shapes and diameters are different within a given cell. To simplify, the form of pores are approximated as a circle and ellipse's these are used as the representation of the adsorbents porosity as shown in figure 4 . 


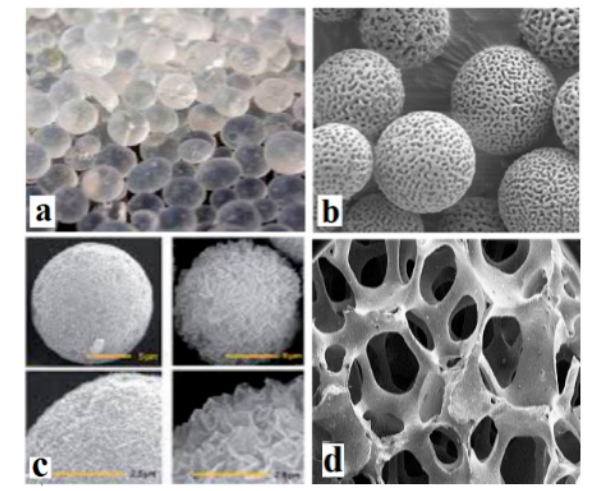

Figure 3. The Internal Structure of porous adsorbent

For one to model a 3D porous structure, a cell modelling method was adapted. This process allowed the $3 \mathrm{D}$ model to mimics the porous fabric of the adsorbent material.

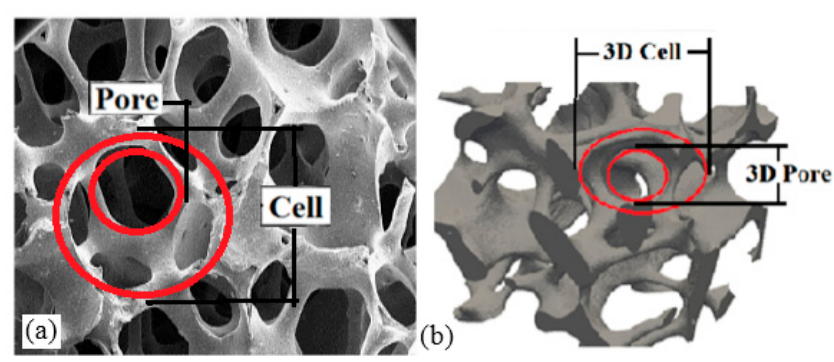

Figure 4 (a) the Internal Structure of porous adsorbent and (b) 3D CAD model of a porous structure with cells

\subsection{Surface Area}

The surface size of a porous adsorbent is defined as the scale of the voids and pores per unit mass $(S)$ or unit bulk volume $\left(S_{V}\right)$ of the porous adsorbent Figure 5. The precise surface based on the porous volume is represented by $S_{O}$.

For instance, the surface of a porous adsorbent made of identical adsorbent spheres of radius $\mathrm{R}$ is:

$S_{v}=\frac{4 \pi R^{2}}{\left(\frac{4}{3}\right) \pi R^{3}}=\frac{3}{R}$

The area of a porous adsorbent is affected by porosity, by the porous adsorbent size and by the shape of the porous adsorbent. Surface plays a significant role in a variety of different application of porous adsorbent. It is the measure of the adsorption capacity of various industrial porous adsorbent; it plays a major role in determining the effectiveness of porous adsorbent. In adsorption cooling systems study, it is associated with the fluid and water vapour conductivity or permeability of porous adsorbent. 


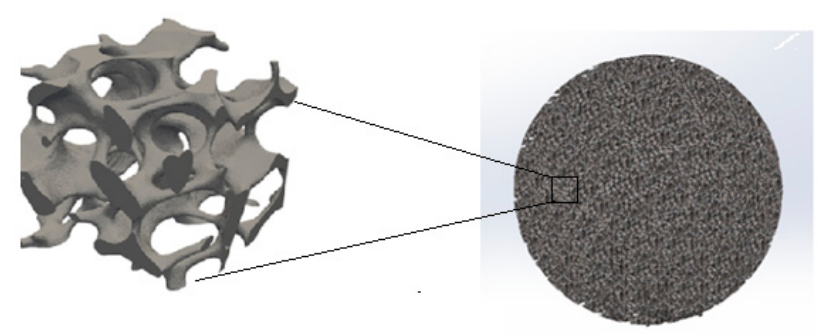

Figure 5 3D modules Surface Area

\subsection{Particle to fluid heat transfer in the adsorption tube}

In the CFD model of the porous adsorbent, the particle-to-fluid heat transfer was simulated by describing all solids (porous spheres, cell wall). The primary value of the resulting heat flux from the porous adsorbent sphere to the water vapour fluid was used $[16,18,19]$.

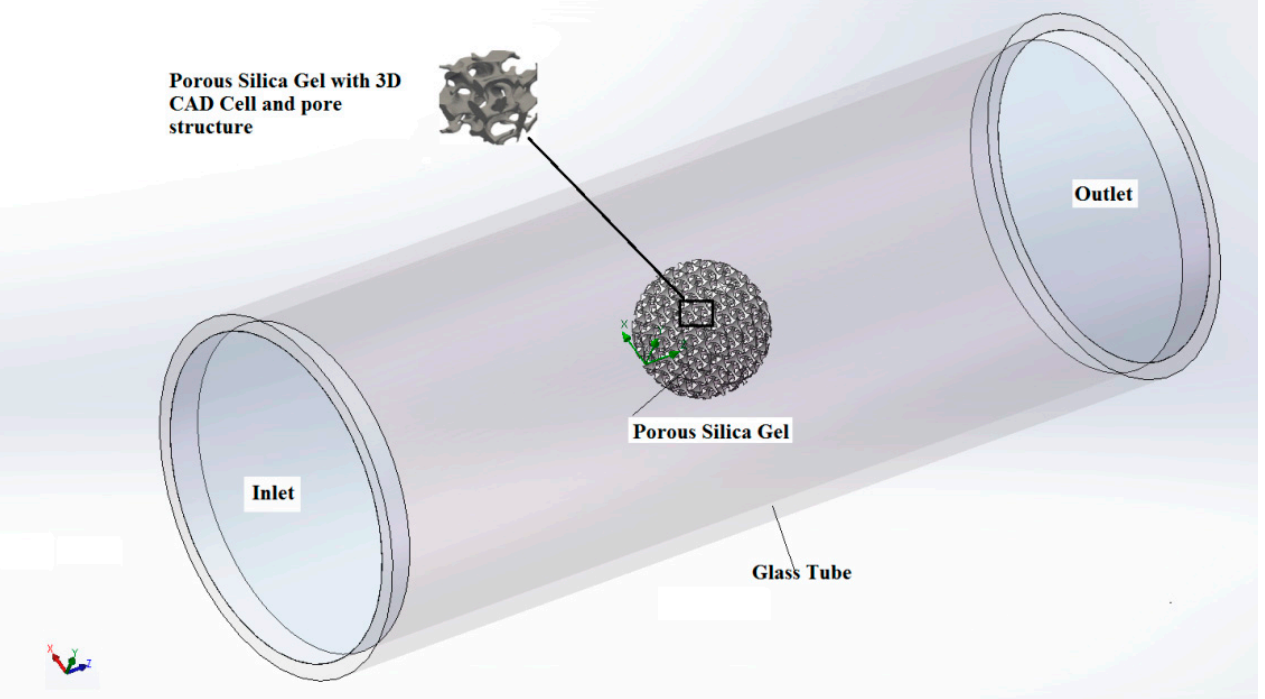

Figure 5 model of the porous adsorbent

\section{2D Velocity profiles}

To study the velocity delivery along the porous adsorbent, some axial and radial cuts were complete along the adsorbent to generate the velocity vector plots. Velocity profiles were observed in the near-wall region of the simulation modelled arrangements $[2,18]$. As was anticipated, in all of the circumstances the analysed, water vapour flow directing took place near the inside wall of the adsorbent, due to the presence of the reserved water vapour flow regions. Sturdy radial flow located in the middle to the wall was also noticeable. This was due to the channelling of the water vapour flow (strong axial vapour flow and reduced radial vapour flow) at the adsorbent bed wall, the local radial heat transfer rate declines, causing a temperature change near the wall. Figures 6 and 7 illustrate the near-wall vapour flow and the velocity contours alongside a transversal cut in the porous adsorbent bed[5,6-20]. 


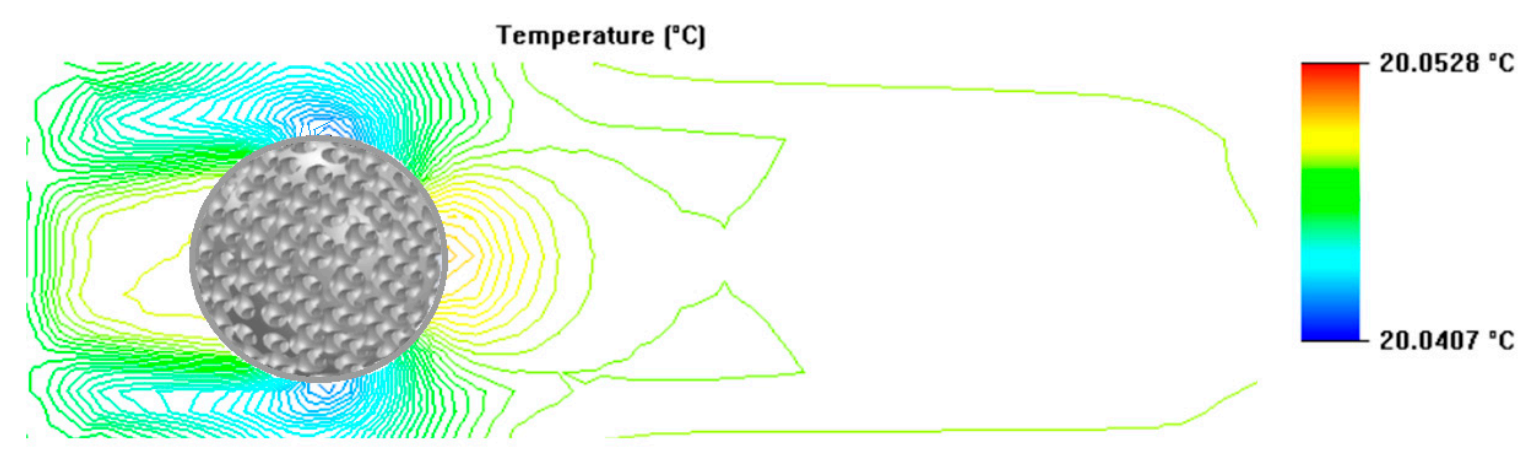

$\operatorname{Min}=20.0407^{\circ} \mathrm{C} \quad \operatorname{Max}=20.0528{ }^{\circ} \mathrm{C}$

Time $=0.164671398 \mathrm{~s}$

Figure 6 Velocity vectors profile in a vertical cross section

\subsection{D Temperature contour plots}

Figure 7 shows temperature contour plots for the CFD simulations to illustrate how the temperature field changed with flow rate. They show a powerful influence on the water vapour flow pattern on temperature profiles. A comparison between the selected CFD simulations shows the increasing kinetic energy inside the porous adsorbent $[3,4,5]$.

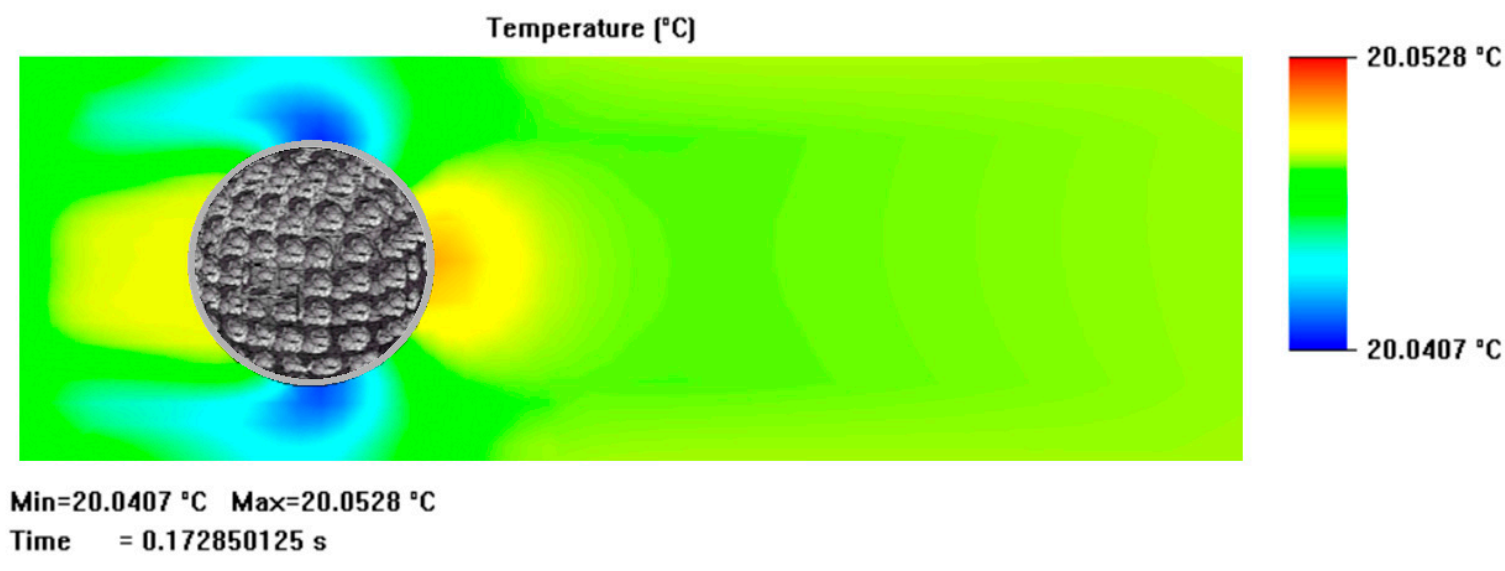

Figure 7 Temperature contour plots

\subsection{Boundary conditions}

Entirely all turbulence CFD simulations model were carried out by allowing for the following boundaries and the resultant boundary conditions. The configuration of the sphere is positioning for the 3-D geometry set-up and the boundaries.

\subsection{Inflow boundary}

Velocity inlet boundary conditions were used to describe the water vapour flow velocity at the inlet boundary (Figure. 13). The water vapour flow velocity ranges from 0.5 to $5 \mathrm{~m} / \mathrm{s}$ to analyse the effects of water vapour inflow rate. The inlet boundary was assumed to have a horizontal velocity contour where a constant inlet air temperature of $4^{\circ} \mathrm{C}$ was specified since it is in the typical temperature range for cold vacuum vapour in the adsorbent bed system [3-18].

The turbulent water vapour flow properties at the inlet boundary were allocated to generate. The essential water vapour turbulence with varying degree of inlet flow turbulence concentrations. In the 
simulation analysis of inlet, vapour flows turbulence effects. Turbulent flow properties at the inlet vapour boundary were enforced to generate the required turbulence at the inlet. To achieve this, some random variations were produced by imposing turbulence length scale and intensity and superimposed on the time averaged stream-wise velocity components at the inlet. The turbulence concentration determined the level of these fluctuations for the specified velocity component at the inlet [1,3-19]. The CFD simulation software enables one to generate the turbulent changes with varying degree of intensity for the same inlet velocity component directly.

\subsection{Outflow boundary}

The CFD simulation of the water vapour, pressure outlet boundary conditions. Were assigned to help describe the static dimension at the outlet boundary. The identified outlet static pressure was set to the value of atmospheric pressure.

\subsection{Wall boundaries}

No-slip velocity boundary conditions were applied to the porous adsorbent sphere and adsorbent bed wall surfaces $(u=v=w=0)$. Constant temperature value of $20^{\circ} \mathrm{C}$ was set at the adsorbent bed surface assuming the porous adsorbents in the adsorbent bed to be at a possible higher temperature and in equilibrium with the adsorbent bed before the adsorption vacuum cooling process started. This temperature was used to make ready the values in the energy equations [15-19].

\subsection{Symmetry boundaries}

Symmetry boundary conditions were applied to the 3D simulation model. Since the 3D model domain was anticipated to be $25 \%$ of the entire simulation solution domain, symmetry boundary conditions were applied at inner cutting surfaces of the vertical plane. Velocity average to symmetry plane and derivatives normal to the symmetry plane of the remaining two velocities were zero. Turbulent properties were enforced regarding turbulence force [5-21].

\subsection{Turbulence models}

Upon completion of CFD simulation domain set-up and assignment of boundary conditions for water vapour velocity, temperature and turbulence properties, CFD simulations were carried out with the use of Reynolds-averaged Navier - Stokes (RANS) equations based two equation vapour turbulence models (the standard $\mathrm{k}$-and the RNG k-3 model). In addition to the turbulence models established, a laminar simulation model was also applied to control the effect of simulation flow modelling method and water vapour turbulence models on the physical behaviour of the flow $[5,16]$. The following section is for the Reynolds averaged based turbulence modelling technique, which was employed to solve the current vapour flow in the present study. Reynolds averaging procedure was applied to the governing equations for resolution of water vapour turbulent flow to obtain the following continuity, Reynolds-averaged momentum and energy equations:

\section{CFD governing equations}

$$
\begin{gathered}
\frac{d \boldsymbol{p}}{\boldsymbol{d} t}+A=\frac{\partial}{\partial x i}(\rho \overline{\mathrm{U}} i)=0 \\
\frac{\partial(\rho \overline{\mathrm{U}} i)}{\partial t}+\frac{\partial}{\partial \mathrm{xj}}(\rho \overline{\mathrm{U}} i \overline{\mathrm{U}} i)=\frac{\partial p}{\partial x i}+\frac{\partial}{\partial x j}\left[\mu \left(\frac{\partial \overline{\mathrm{U}} i}{\partial x j}+\frac{\partial \overline{\mathrm{U}} j}{\partial x i}-\frac{2}{3} \delta i j \frac{\partial \overline{\mathrm{U}} l}{\partial x l}\right.\right.
\end{gathered}
$$




$$
\begin{aligned}
& +\frac{\partial}{\partial x j}(-\rho \bar{U} i \bar{U} j) \\
& \frac{\partial(\rho e)}{\partial t}+\frac{\partial}{\partial x j}[u j(p e+p)]=\frac{\partial}{\partial x j}\left(k e f \frac{\partial T}{\partial x j}+u i(\tau i j) e f\right)+S h
\end{aligned}
$$

The stress $(\tau \mathrm{ij})$ representing the viscous heating including turbulence effects is given by

$$
(\tau \mathrm{ij}) \mathrm{ef}=(\mu+\mu t)\left[\frac{\partial u i}{\partial x j}+\frac{\partial u j}{\partial x i}-\delta i j \frac{\partial \overline{\mathrm{U}} l}{\partial x l}\right]-\frac{2}{3} p k \delta i j
$$

where $k$ is the resolved turbulence kinetic $(k)$ energy:

$$
\mathrm{k}=\frac{1}{2} u^{\prime} i u^{\prime} i
$$

In an eddy-viscosity simulation model, the Reynolds stresses are assumed to be related to the mean vapour velocity gradients with the constant proportionality being the eddy viscosity $\mu_{t}$. This hypothesis is known as Boussinesq eddy viscosity hypothesis and provides the following expression for the Reynolds stresses (Hinze, 1975):

$$
-\rho \overline{u_{i}^{\prime} u_{j}^{\prime}}=\frac{2}{3} \rho k \delta_{i j}+\mu_{t}\left(\frac{\partial \bar{u}_{i}}{\partial x_{j}}+\frac{\partial \bar{u}_{j}}{\partial x_{i}}\right)+\frac{2}{3} \mu_{t} \frac{\partial \bar{u}_{j}}{\partial x_{j}} \delta_{i j}
$$

The eddy viscosity, ${ }^{\mu_{t}}$ in Eq. (6) was acquired by supposing that it was proportional to the product of a turbulent velocity and length scale.

\section{The $\mathrm{k}-\mathbf{3}$ turbulence model}

Isotropic eddy viscosity based standard $k-3$ turbulence simulation model was employed for the vapour turbulence computations. In this simulation model, velocity and length scales characterising turbulent vapour field were obtained from two parameters: turbulent kinetic energy, $k$ and dissipation $\varepsilon$. The velocity scale was $\sqrt{k}$ and the length scale was ${ }^{\sqrt{k^{3}}} / \varepsilon$. Therefore, the turbulent vapour viscosity, ${ }^{\mu_{t}}$, in Eq. (6) was simulated by merging $\mathrm{k}$ and $\varepsilon$. as

$$
\mu_{\mathrm{t}}=\rho \mathrm{C}_{\mu} \frac{k^{2}}{\varepsilon}
$$

The standard $k-\varepsilon$ turbulence simulation model, initially developed by Launder and Spalding (1972), is a semi-empirical model based on turbulence transport equations for the turbulent kinetic energy, $\mathrm{k}$ and its dissipation rate, $\varepsilon$. The turbulence transport equations $k$ were derived from the exact equation while the turbulence transport equation for $\varepsilon$ was obtained using the physical reasoning. The water vapour turbulent kinetic energy $k$ and its rate of dissipation rate $\varepsilon$ were achieved from the following two separate transport equations given by Launder and Spalding (1972) 


$$
\begin{aligned}
& \frac{\partial}{\partial t}(\rho k)+\frac{\partial}{\partial x_{i}}\left(\rho \bar{u}_{i} k\right)=\frac{\partial}{\partial x_{i}}\left[\left(\mu+\frac{\mu_{t}}{\sigma_{k}}\right) \frac{\partial k}{\partial x_{i}}\right]+G_{k}-\rho \varepsilon \\
& \frac{\partial}{\partial t}(\rho \varepsilon)+\frac{\partial}{\partial x_{i}}\left(\rho \bar{u}_{i} \varepsilon\right)=\frac{\partial}{\partial x_{i}}\left[\left(\mu+\frac{\mu_{t}}{\sigma_{\varepsilon}}\right) \frac{\partial \varepsilon}{\partial x_{i}}\right]+C_{1} \frac{\varepsilon}{k} G_{k}-C_{2 x} \rho \frac{\varepsilon^{2}}{k}
\end{aligned}
$$

In these equations, $G_{k}$ represents the generation of turbulent kinetic energy due to the mean velocity gradients:

$$
G_{k}=-\rho \overline{u_{i}^{\prime} u_{j}^{\prime}} \frac{\partial \bar{u}_{j}}{\partial x_{i}}
$$

The corresponding default values for the given coefficients are $\mathrm{C}_{1 \varepsilon}=1.44, \mathrm{C}_{2 \varepsilon}=1.92, \mathrm{C}_{\mu}=0.09, \sigma_{\mathrm{k}}=1.0$, and $\sigma_{\varepsilon}=1.0$.

The renormalized group (RNG) $\mathrm{k}-\varepsilon$ turbulence model Yakhot and Orszag (1986) proposed another k$\varepsilon$ turbulence model variant where the standard model enhanced the performance characteristics. The RNG $\mathrm{k}-\varepsilon$ turbulence model was based on renormalized group theory. This $3 \mathrm{D}$ model is parallel in form to the standard turbulence model.

However, the RNG turbulence model differs from the standard model by inclusion of an additional sink term in the turbulence dissipation equation. This was to account for non-equilibrium strain rates and employs different values for various model coefficients. In this example, the form of $\mathrm{k}$ equation remains same. The turbulence dissipation equation of the RNG $\mathrm{k}-\varepsilon$ turbulence model includes the following sink term:

$$
\frac{C_{\mu} \rho \eta^{3}\left(1-\eta / \eta_{0}\right)}{1+\beta \eta^{3}} \frac{\varepsilon^{2}}{k}
$$

Where $\eta_{0}=4.38$ and $\beta=0.012$. The above sink term also employs a parameter, $\eta$ ratio of the characteristic time scales of turbulence and low flow field:

$$
\eta=\frac{S k}{\varepsilon}
$$

Where $\mathrm{S}=\sqrt{2 \mathrm{~S}_{\mathrm{ij}} \mathrm{S}_{\mathrm{ij}}}=\sqrt{\mathrm{G}_{\mathrm{k}} / \mu_{\mathrm{t}}}$ The first model coefficients of the RNG $\mathrm{k}-3$ turbulence model are $\mathrm{C}_{\mu}, \mathrm{C}_{1 \varepsilon}, \mathrm{C}_{2 \sigma}, \sigma_{\mathrm{k}}, \sigma_{\varepsilon}$ and Von Karman constant $k$. Recommended values for these model.

$$
\begin{aligned}
& \text { coefficients are } C_{1 \varepsilon}=1.41, C_{2 \varepsilon}=1.68, C_{\mu}=0.085, \sigma_{k}=0.7179 \text {, } \\
& \sigma_{\varepsilon}=0.7179 \text { and } \kappa=0.3875
\end{aligned}
$$

\section{Adsorption-Desorption Simulation Models}

There remain two main types of adsorption: chemical adsorption and Physical adsorption. Physical sorption occurs through van der Waals forces while chemical adsorption involves covalent chemical bonds. In porous adsorbents that adsorb mainly gas or water vapour 
under a vacuum use Physical adsorption. As a result, this is the type of adsorption that will discourse in this paper $[16,17,18]$.

The most common description of Physical adsorption in homogeneous porous adsorbents is through the Brunauer, Emmett and Teller (BET) isotherm. The theory for the (BET) isotherm is based on the following assumptions:

1. Gas molecules physically adsorb on a porous adsorbent in layers infinitely;

2. There is no interface between each adsorption level

3. The Langmuir theory can be applied to each adsorption layer.

The resultant BET

$$
\frac{1}{v\left[\left(p_{0} / p\right)-1\right]}=\frac{c-1}{v_{\mathrm{m}} c}\left(\frac{p}{p_{0}}\right)+\frac{1}{v_{m} c}
$$

Where $p$ and $T_{0}$ are the saturation vapour pressure of water vapour at the temperature of adsorption, $v$ is the adsorbed vapour amount and $v_{m}$ is the monolayer adsorbed water vapour amount. $c$ is the BET continuous,

$$
c=\exp \left(\frac{E_{1}-E_{\mathrm{L}}}{R T}\right)
$$

Where $E_{1}$ is the adsorption heat for the first adsorption layer, and $E_{\mathrm{L}}^{*}$ is that for the second adsorption layers and higher it is equivalent to the heat of liquefaction.

\section{Discussion and Validation Results}

\subsection{Experimental Apparatus Description}

There are many methods used for measuring the adsorption uptake of vapour by adsorbent one of the procedures are the (DVS) Dynamic vapour sorption process. This system is designed to measure adsorption/desorption of porous media materials see figure. 8 for Schematic layout of the of the (DVS) instrument [10-21]. 


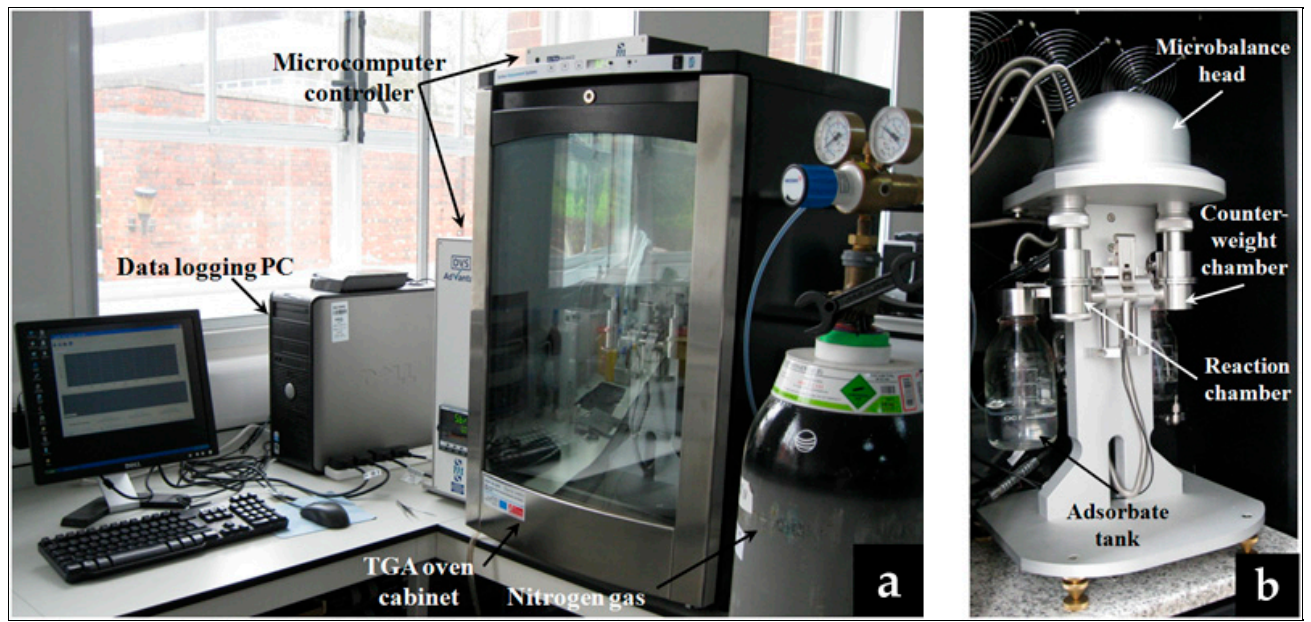

Figure 8 pictographic view of the used DVS apparatus. This arrangement measures the change in weight of a porous adsorbent sample as heated for desorption of water vapour and cooled for the adsorption of water vapour or held at a constant temperature.

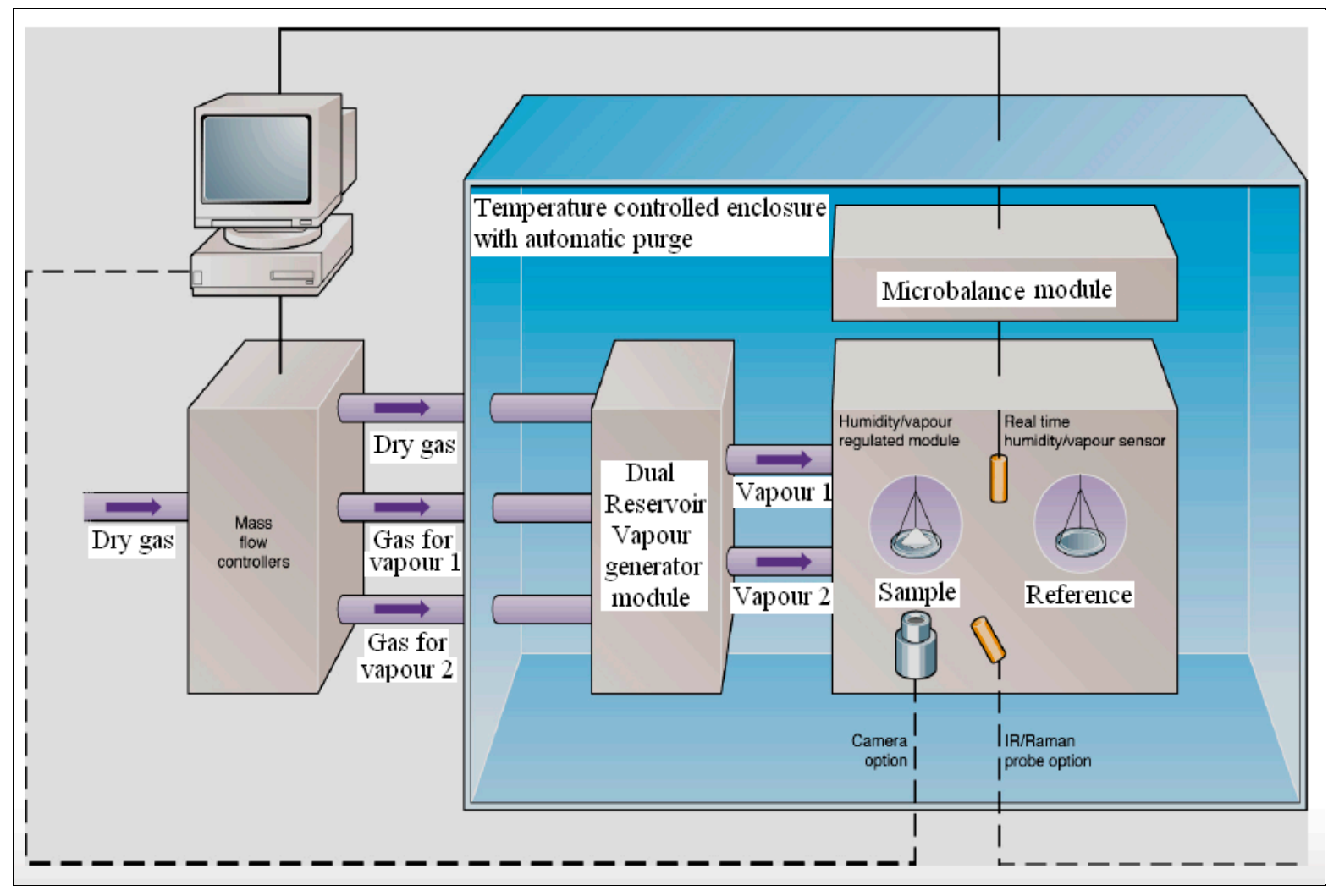

Figure 9 Schematic of the (DVS) instrument

\section{Setup and Procedure}

The Dynamic Vapour Sorption (DVS) technique are used for adsorption experimentations DVS gives a direct measurement of the quantity of water vapour adsorbed throughout the adsorption uptake process. In the DVS, the sample containers are suspended on an extension wire that is connected to the microbalance see figure 10 . 

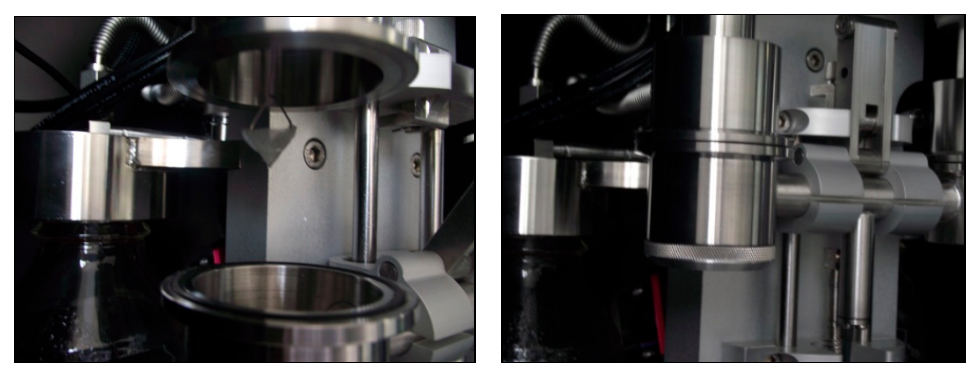

Figure 10 sample container suspended on an extension cable.

The microbalance measures the weight of the sample, and it can detect pressure changes of the sample[10,18-21].
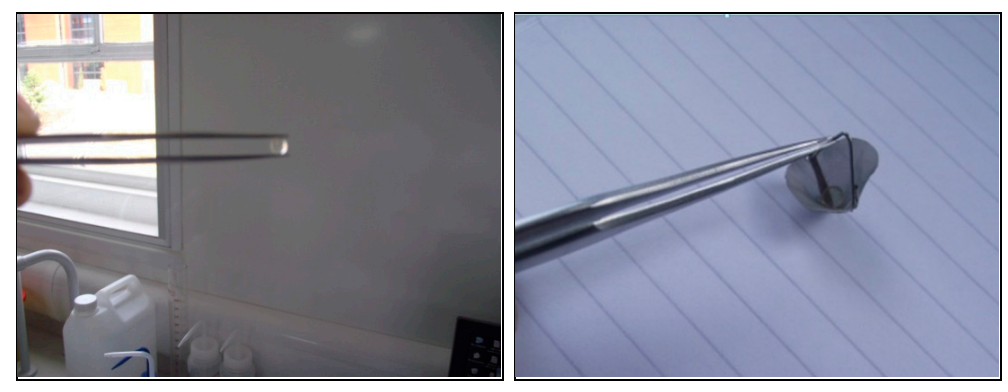

Figure 11 Silica gels size $5 \mathrm{~mm}$ type A, B and RD

\section{Results}

\subsection{The Isotherm Plot for Silica Gel Size 5mm}

In figure 12 the isotherm plot is for silica gel size $5 \mathrm{~mm}$. The plot begins at water vapour performance proximate zero and $1{ }^{\circ} \mathrm{C}$. The outline and location of the isotherm hysteresis give you the information about the adsorption method and sample porosity. The temperature of water vapour in the adsorbent sample chamber is a continuous $25^{\circ} \mathrm{C}$. The isotherm plot is used to support the desorption rates of the porous adsorbent. The isotherm also displays whether the porous adsorbent hysteresis and what impact it will have on water vapour activity after desorption to a given moisture content drying curve, for silica gel type B, size $5 \mathrm{~mm}$. Figure 12., displays two isotherms, one obtained by adsorption of water vapour from the porous adsorbent sample from a waterless state and the other acquired by desorption of water vapour from the porous adsorbent sample.

The water vapour adsorption is shown (red), and water vapour desorption is presented (blue), isotherms at a temperature of $25^{\circ} \mathrm{C}$. The isotherm plot characterises the limits or bounding isotherms since they begin at water performance near zero and one-degree centigrade. The shape of the isotherm hysteresis gives the information about the adsorption mechanism and sample porosity of the $5 \mathrm{~mm}$ silica gel. 


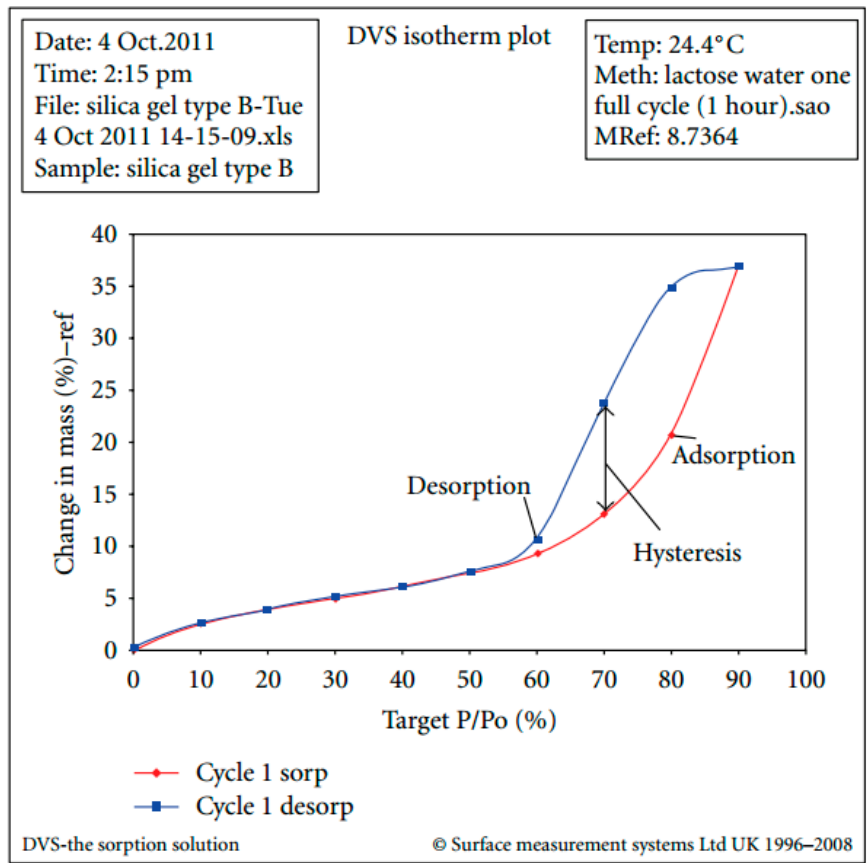

Figure.12 water vapour adsorption performance of porous adsorbent type B size $5 \mathrm{~mm}$ at $25^{\circ} \mathrm{C}$ one cycle experiment.

\subsection{D Results from the porous adsorbent simulation model}

First, the effects of model parameters on the behaviour of adsorption and desorption isotherms were studied. Figure 13 shows the characteristic of the water adsorption and desorption isotherm. All parameters are static except the parameter representing the adsorption into the micropore, from the graphs has an increase in adsorption.

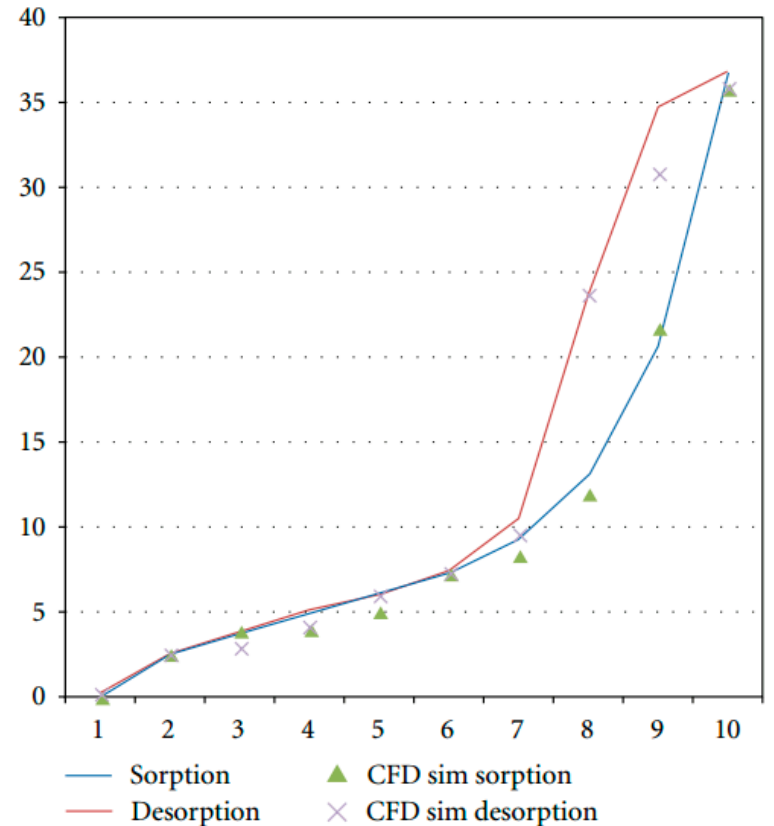

Figure 13 Experiment Vs CFD simulations 


\section{Validations}

Since the CFD methodology, it is necessary to verify if the simulated simulation results are valid. Even though the CFD simulation code are based on the fundamental principles of fluid flow and heat transfer, some of the boundary problems are simulated using experimental output results not necessarily appropriate for the fixed bed application. In this section, a performed validation study is presented to validate the used CFD simulation code and the used boundary models for use in porous adsorbent packed fixed beds. In this section, the intent is to show that a validation of CFD simulation porous adsorbent in fixed bed geometries was performed. A short explanation of the experimental and CFD simulation approaches will be given. Additionally, some of the results and discussion will be presented.

\section{Conclusion}

The literature analysis, CFD simulation and experimental results intended to provide a fundamental understanding of porous adsorbent bed fundamentals. The initial stages of the investigation focused on the general analysis of low aspect ratio, Porosity variation and heat transfer in porous adsorbent packed beds. The study found that numerous authors had examined low aspect ratio adsorbent packed beds. It was found that CFD simulation allowed the researcher to simulate randomly packed 3D beds and with the growth in computational power in recent years it was possible to use CFD codes successfully on the simulation of porous adsorbent beds. The contact points, mesh and turbulence models were considered to be critical to the accuracy of the CFD simulations. The drop pressure study found that that correction of the Ergun equation for the wall effect was the most promising correlation to correlate pressure drop for Reynolds numbers. The by doing the velocity simulation it was found that an increase in porosity at the adsorbent bed wall region changes the velocity circulation affecting the higher velocities at the near-wall regions. The CFD simulation, therefore, provided the fundamental understanding of the important factors influencing porous adsorbent packed beds.

\section{References}

[1] Bertil Andersson, K.E. (1961) "Pressure drop in ideal fluidization," Chemical Engineering Science, vol. 15, no. 3-4, pp. 276-297

[2] Ergun, S. (1952) "Fluid flow through packed columns," Chemical Engineering Progress, vol. 48, pp. 89-94, 1952

[3]Molerus, O., and Schweinzer, J. (1989) "Resistance of particle beds at Reynolds numbers up to Re $\approx 104$," Chemical Engineering Science, vol. 44, no. 5, pp. 1071-1079

[4] Dixon, A.G., and Cresswell, D. L. (1986) "Effective heat transfer parameters for transient packedbed models," AIChE Journal, vol. 32, no. 5, pp. 809-819

[5] Rahimi, M., and Mohseni, M. (2008) "CFD modelling of the effect of absorbent size on absorption performance of a packed bed column," Korean Journal of Chemical Engineering, vol. 25, no.3, pp. $395-401$

[6] Klaus Schnitzlein (1993) Prediction of velocity profiles in packed beds, Journal of Chemical Engineering, Volume 48, Pages 811-815.

[7] Akahira, A., Amanul Alam, K.C., YoshinoriHamamoto (2005), "Experimental investigation of mass recovery adsorption refrigeration cycle," International Journal of Refrigeration, vol. 28, no. 4, pp. $565-572$ 
[8] M.D. Kelleher, R.K. Shah, K.R. Sreenivasan, Y. Josh (1993) Experimental Heat Transfer, Fluid Mechanics and Thermodynamics, Volume 1

[9] Chang, K.S., Chen, M.T., Chung, T.W. ( 2005) "Effects of the thickness and particle size of silica gel on the heat and mass transfer performance of a silica gel coated bed for air-conditioning adsorption systems," Applied Thermal Engineering, vol. 25, no. 14-15, pp. 2330-2340

[10] Liou, May-Fun. (2005) A numerical study of transport phenomena in porous media, PhD thesis, Case Western Reserve University,2005

[11] Mueller, G.E. (1992) "Radial void fraction distributions in randomly packed fixed beds of uniformly sized spheres in cylindrical containers," Powder Technology, vol. 72, no. 3, pp. 269-275

[12] Kopanidis, K., and Theodorakakos, A. (2008) "Numerical Simulation of Fluid Flow and Heat Transfer with Direct Modelling of Microscale Geometry," in Proceedings of the 5th

EuropeanThermal-Sciences Conference, The Netherlands, 2008

[13]Anikeenko, A.V., Medvedev, N.N., Kovalev, M.K., and Melgunov M.S. (2009), "Simulation of gas diffusion in porous layers of varying structure," Journal of Structural Chemistry, vol. 50, no. 3, pp. $403-410$

[14]Wang, D.C., et. al. (2005) "Study of a novel silica gel - water adsorption chiller: Part II. An experimental study," International Journal of Refrigeration, vol. 28, no. 7, pp. 1084-1091

[15] Augier, F., Laroche C., and Brehon, E. (2008) "Application of computational fluid dynamics to fixed bed adsorption calculations: effect of hydrodynamics at laboratory and industrial scale," Separation and Purification Technology, vol. 63, no. 2, pp. $466-474$

[16]White, J. (2012)“Computational Fluid Dynamics Modelling and Experimental Study on a Single Silica Gel Type B", Modelling and Simulation in Engineering, Hindawi Publishing Corporation Published online January 2012 vol. 2012, Article ID598479, nine pages.

[17]White, J. (2012) "A CFD Simulation on How the Different Sizes of Silica Gel Will Affect the Adsorption Performance of Silica Gel”, Modelling and Simulation in Engineering, Hindawi Publishing Corporation Published online January 2012vol. 2012, Article ID 651434, 12 pages.

[18] White, John (2013) CFD simulation of silica gel and water adsorbent beds used in adsorption cooling system PhD Thesis, University of Birmingham.

[19] White, John (2016) CFD Simulation and Experimental Analyses of a Copper Wire Woven Heat Exchanger Design to Improve Heat Transfer and Reduce the Size of Adsorption Beds by the authors Computation, doi:10.3390/computation4010008

[20] Jing Zhu and Chunmei Qiu, (2014) A microfluidic device for multiple single-nucleotide polymorphism genotyping.

[21] Ahmed S. Alsamana, Ahmed A. (2016) A state of the art of hybrid adsorption desalinationcooling systems Renewable and Sustainable Energy Reviews Volume 58, May, Pages 692-703

[22] A Sharafian, C McCague, M Bahrami (2015) Impact of fin spacing on temperature distribution in adsorption cooling system for vehicle A/C applications - International Journal of Refrigeration, Elsevier.

(c) 2016 by the authors; licensee Preprints, Basel, Switzerland. This article is an open access article distributed under the terms and conditions of the Creative Commons by Attribution (CC-BY) license (http://creativecommons.org/licenses/by/4.0/). 